\title{
Global Pediatric Pulmonology Alliance recommendation to strengthen prevention of pediatric seasonal influenza under COVID-19 pandemic
}

\author{
Kun-Ling Shen ${ }^{1} \cdot$ Leyla Namazova-Baranova ${ }^{2} \cdot$ Yong-Hong Yang ${ }^{3} \cdot$ Gary Wing Kin Wong $^{4} \cdot$ Lanny J. Rosenwasser $^{5}$. \\ Lance E. Rodewald ${ }^{6}$. Anne Eng Neo Goh ${ }^{7}$. Eitan Kerem ${ }^{8}$. Chris O'Callaghan ${ }^{9} \cdot$ T. Bernard Kinane $^{10}$. Basil Elnazir ${ }^{11}$. \\ Rina Triasih ${ }^{12} \cdot$ Rosemary Horne $^{13} \cdot$ Anne B. Chang $^{14} \cdot$ Jim Buttery $^{15} \cdot$ Ruth A. Etzel $^{16} \cdot$ Kazunobu Ouchi $^{17}$. \\ Hilary Hoey ${ }^{18}$. Varinder Singh ${ }^{19}$. Genesis C. Rivera ${ }^{20}$. Spencer S. $\mathrm{Li}^{21} \cdot \mathrm{Yu}_{\mathrm{Guan}}{ }^{21}$ on behalf of the Global Pediatric \\ Pulmonology Alliance (GPPA) Council · Ling Cao ${ }^{22} \cdot$ Yue-Jie Zheng ${ }^{3} \cdot$ Lu-Zhao Feng $^{23} \cdot$ Wu Zhong ${ }^{24} \cdot$ Zheng-De Xie $^{25}$.

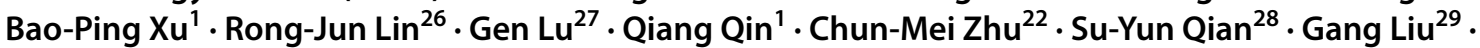 \\ Cheng-Song Zhao ${ }^{29}$. Zhuang Wei ${ }^{30} \cdot$ Yu-Hong Zhao ${ }^{31}$ on behalf of the Global Pediatric Pulmonology Alliance \\ (GPPA) Expert Panel on Infectious Diseases \& COVID-19
}

Received: 6 August 2020 / Accepted: 19 August 2020 / Published online: 13 September 2020

(c) Children's Hospital, Zhejiang University School of Medicine 2020

The World Health Organization (WHO) announced COVID19 as a global pandemic in March of 2020 [1]. The COVID19 pandemic may persist for a long period of time. Global prevention and control becomes a complex and challenging task, and such efforts should be sustained. Although general measures, such as social distancing, face masks, respiratory hygiene and hand sanitization, will bear fruits for decreasing spread of other respiratory illnesses including influenza, the specific prevention through vaccination is a key focus especially in the upcoming winter and spring seasons [2].

Winter and spring are the peak seasons for influenza and other common respiratory infectious diseases. Children are a high-risk group for influenza. The prevalence of influenza in children is $20-30 \%$ every year [3], and it often enhances outbreaks in the community. The pediatric population in child care centers, kindergartens and primary schools is closely confined and is vulnerable to mass outbreaks [4]. The high infection rate of influenza in children is also one of the important reasons for the spread of influenza virus in the community [5]. If an outbreak of influenza coincides with that of COVID-19, the impact on the pediatric population will be even more severe [6].

According to WHO, the COVID-19 outbreak in early 2020 occurred at the same time as the influenza outbreak [7], and the primary clinical manifestations of influenza and COVID-19 overlap considerably. An epidemic of influenza

Yong-Hong Yang

yyh628628@sina.com

Extended author information available on the last page of the article would challenge the diagnosis and treatment in fever clinics and the supplies of personal protective equipment (PPE). Therefore, the upcoming winter and spring seasons are of high alert [8], as immense pressure will be placed on hospitals' overall diagnosis and treatment capacity and on their critical care capacity. Co-infection of influenza and COVID19 can occur in patients and present with severe symptoms. This will further increase the complexity of clinical diagnosis and treatment [6]. Hospitals usually have concentrated patient flows, and the risk of cross infection is increased, which threatens the health of all patients, their caregivers, and medical staff members.

The WHO, the United Nations International Children's Emergency Fund (UNICEF), and various nations have called for strengthening of prevention and control of seasonal influenza while making every effort to cope with the COVID-19 pandemic. These organizations vigorously advocate influenza vaccination as the primary measure for pandemic prevention and control, so as to minimize the interference of an influenza epidemic during the prevention and control of the COVID-19 pandemic situation. According to the WHO recommendation, influenza vaccination is an effective supplementary measure against the COVID-19 pandemic for countries that are about to enter the winter. All eligible individuals should receive the influenza vaccine [2]. UNICEF calls for influenza vaccination for children during the COVID-19 pandemic to protect them from other diseases [9]. The US Centers for Disease Control (CDC) stated that in the coming winter influenza and COVID19 may spread simultaneously and recommended vaccination for all children older than 6 months [10]. The Australian government strongly recommends that people, especially children 
and other high-risk groups, should be vaccinated with influenza vaccine when actively coping with the possible overlap of COVID-19 and influenza [11]. The British public health agency also stated that owing to the COVID-19 outbreak, influenza vaccination is more important than ever before [12]. New Zealand called for vaccination for high-risk target populations [13] (such as children, seniors and pregnant women) to protect communities and to utilize resources most effectively. Brazil initiated early vaccination to reduce influenza cases in the midst of COVID-19, with children from 6 months to 6 years of age being the priority target population [14]. Thus, preschool-age and younger children are the key vaccination population, and influenza vaccination is the best preventive measure.

\section{Recommendations}

To control the pandemic situation more effectively, the Global Pediatric Pulmonology Alliance (GPPA), based on best-practices and evidence from various countries, recommends that all global and regional organizations, medical societies, and health agencies join hands in the prevention of seasonal influenza in children. Measures to improve influenza vaccination include promoting public awareness about infection control measures and thorough education of the benefit of influenza vaccination. The ultimate goal is to achieve early notification, early appointment and early vaccination, which will reduce the likelihood of coincident epidemics of influenza and COVID-19 this winter. It is recommended that vaccination policy, vaccine safety, and medical advice be promoted and reiterated in child care centers, kindergartens, and schools [15]. Countries and regions with ample resources should implement free influenza vaccination in child care centers, kindergartens and schools, so as to increase the coverage rate of influenza vaccination among the pediatric population [16]. It is also advised that in countries or regions, where influenza vaccination may not be part of national immunization program (NIP), pediatricians should check with respective health authorities or local guidelines to reach the suggested beneficiary population.

\section{Strategies in influenza vaccination}

\section{(1) Infants under 6 months}

Influenza vaccine cannot be given to infants under 6 months. We recommend parents, caregivers, and family members of the infants to be vaccinated [4], and if included in national recommendations, expectant mothers should receive influenza vaccination during pregnancy to protect themselves and protect newborns from influenza infection during their first six month of life.
(2) Children over 6 months and school-age children

This is the priority population for influenza vaccination. All children over 6 months of age are eligible, except for those who are allergic to vaccine substances/components or who are experiencing fever or an acute infection. Children with a history of egg allergy of any severity can receive any licensed, recommended, and age-appropriate influenza vaccine. Children with stable chronic pulmonary, cardiovascular, renal or neurological diseases should get the influenza vaccine as early as possible [4]. Children with primary immunodeficiency, HIV infection and secondary immunodeficiency (leukemia, carcinoma, receiving immunosuppressive therapy, etc.) should consult with medical professionals before using live attenuated influenza vaccines.

\section{Schools and kindergartens}

Staff should be vaccinated every year [17]. Schools and kindergartens should carry out awareness and education campaigns on prevention and control of infectious diseases and on promotion of vaccination to students and parents, so as to improve vaccination rates and to prevent the outbreak of clustering epidemic [17, 18]. Family members of children and frequent visitors (such as caregivers, housekeeping workers, etc.) also should be vaccinated annually [17].

\section{Pediatric health care workers}

Pediatric medical staff and workers are in frequent and close contact with children. They should be vaccinated every year. This should also be considered for other staff groups, such as hospital cleaners and porters. This protects the pediatric health care workers, maintains health care capacity by reducing sick leave, and also helps to reduce transmission from members of staff to children and others, thereby reducing the risk of hospital-acquired infections. It is a concern that shedding of influenza virus has been shown shortly before clinical illness develops and that health care workers frequently continue to work while unwell.

\section{Vaccination schedule}

Influenza vaccine needs to be given every year. Countries and regions around the world should prepare ample vaccine supplies and should complete vaccination of the pediatric population about 2-3 months before the arrival of the influenza season [4]. Although the GPPA is advocating vaccinations (as described above), public health priorities may differ across nations; therefore, pediatricians should check their national guidelines for the eligible population, available vaccine products and the number of doses to 
administer based on age, vaccination history, and vaccine product to make a final call.

\section{Vaccination methods}

The inactivated vaccine is to be injected intramuscularly (except intradermal injection preparation). Children over 1 year and adults should be vaccinated in the deltoid muscle of the upper arm as the first choice. The anterolateral thigh is the best vaccination site for infants between 6 months and 1 year. Patients with thrombocytopenia or other hemorrhagic diseases may have the risk of bleeding during intramuscular injection; so subcutaneous injection is recommended. Injection dosage should be referenced to the product specification.

\section{Precautions for vaccination under COVID-19 pandemic}

(1) Parents should make an appointment before vaccination and arrive at the vaccination site on time, so as to avoid crowding at the site.

(2) Parents or individuals accompanying children for vaccination should wear protective masks (such as surgical masks) and keep a physical distance of more than $1 \mathrm{~m}$ ( $1.8 \mathrm{~m}$ is required in the US) from others.

(3) Parents or individuals accompanying children should use face masks and should follow strict hand hygiene and routine disinfection according to instructions of medical staff before vaccination.

(4) After vaccination, parents should take the child to a designated area for routine observation and then go home immediately to avoid a prolonged stay in the facility or other public areas.

(5) Children and their parents or caregivers should apply hand hygiene and should dispose of face masks properly after returning home.

(6) After returning home, parents should observe the overall health of the child, keep his/her vaccination spot clean, and watch for possible complications of vaccination.

(7) Expert advice should be available to support vaccination staff in answering questions about vaccine eligibility.

In conclusion, under the COVID-19 pandemic, getting the influenza vaccine in time offers children a safer winter and a healthier future.

Author contributions Feng LZ, Zhong W and Xie ZD contributed to data curation; all the other authors wrote a part of original draft.

\section{References}

1. World Health Organization. WHO Director-General's opening remarks at the media briefing on COVID-19. 2020. https://www. who.int/dg/speeches/detail/who-director-general-s-opening-remar ks-at-the-media-briefing-on-covid-19---11-march-2020. Accessed 31 July 2020.

2. World Health Organization. WHO Director-General's opening remarks at the media briefing on COVID-19. 2020. https://www. who.int/dg/speeches/detail/who-director-general-s-opening-remar ks-at-the-media-briefing-on-covid-19---27-april-2020. Accessed 31 July 2020.

3. WHO. Vaccines against influenza WHO position paper. Wkly Epidemiol Rec. 2012;87:461-76.

4. National Immunization Advisory Committee (NIAC) Technical Working Group (TWG), Influenza Vaccination TWG. Technical guidelines for seasonal influenza vaccination in China, 20192020. Chin J Epidemiol. 2019;40:1333-499.

5. Uscher-Pines L, Schwartz HL, Ahmed F, Zheteyeva Y, Tamargo Leschitz J, Pillemer F, et al. Feasibility of social distancing practices in US schools to reduce influenza transmission during a pandemic. J Public Health Manag Pract. 2020;26:357-70.

6. Wu X, Cai Y, Huang X, Yu X, Zhao L, Wang F, et al. Co- infection with SARS-CoV-2 and Influenza A virus in patient with pneumonia. China Emerg Infect Dis. 2020;26:1324-6.

7. World Health Organization. Influenza laboratory surveillance information by the Global Influenza Surveillance and Response System (GISRS). 2020. https://www.who.int/influenza/gisrs laboratory/updates/flunet_globalviruscirculation_20200508.pdf. Accessed 31 July 2020.

8. World Health Organization. Recommended composition of influenza virus vaccines for use in the 2020-2021 northern hemisphere influenza season. 2020. https://www.who.int/influenza/vaccines/ virus/recommendations/202002_recommendation.pdf?ua $=1$. Accessed 31 July 2020.

9. United Nations International Children's Emergency Fund. Vaccinations and COVID-19: What parents need to know. 2020. https ://www.unicef.org/coronavirus/vaccinations-and-covid-19-whatparents-need-know. Accessed 31 July 2020.

10. Centers for Disease Control and Prevention. Frequently asked influenza (flu) questions: 2020-2021 season. 2020. https://www. cdc.gov/flu/season/faq-flu-season-2020-2021.htm. Accessed 31 July 2020.

11. Tasmanian Government. Influenza vaccination. 2020. https:// coronavirus.tas.gov.au/keeping-yourself-safe/what-you-can-do/ influenza-vaccination. Accessed 31 July 2020.

12. NHS England and NHS Improvement. The national flu immunisation programme 2020/21. 2020. https://assets.publishing.servi ce.gov.uk/government/uploads/system/uploads/attachment_data/ file/885281/The_national_flu_immunisation_programme_2020_ to_2021.pdf. Accessed 31 July 2020.

13. The official website of the New Zealand Government. Govt begins largest ever Flu vaccine campaign early. 2020. https://www.beehi ve.govt.nz/release/govt-begins-largest-ever-flu-vaccine-campaignearly Accessed 31 July 2020.

14. Por Vanessa Aquino, da Agência Saúde.comeca-segunda-feiravacinacao-contra-gripe. 2020. https://www.saude.gov.br/notic ias/agencia-saude/46567-comeca-segunda-feira-vacinacao-contr a-gripe. Accessed 31 July 2020.

15. Communicable Diseases Network Australia. CDNA national guidelines for public health units:seasonal influenza infection. 2020. https://www1.health.gov.au/internet/main/publishing.nsf/ Content/cdna-song-influenza.htm. Accessed 31 July 2020. 
16. Weinberg GA. Nontraditional Uses of live attenuated influenza vaccine: School-Located influenza vaccination. J Pediatric Infect Dis Soc. 2020;9(Suppl 1):S19-23.

17. Communicable Diseases Network Australia. Guidelines for the prevention, control and public health management of influenza outbreaks in residential care facilities in Australia. 2020. https:// www1.health.gov.au/internet/main/publishing.nsf/Content/cdnaflu-guidelines.htm. Accessed 31 July 2020.
18. Committee on Infectious Diseases. Recommendations for prevention and control of influenza in children, 2018-2019. Pediatrics. 2018;2018(142):e20182367.

Publisher's Note Springer Nature remains neutral with regard to jurisdictional claims in published maps and institutional affiliations.

\section{Affiliations}

Kun-Ling Shen ${ }^{1} \cdot$ Leyla Namazova-Baranova ${ }^{2}$. Yong-Hong Yang ${ }^{3} \cdot$ Gary Wing Kin Wong $^{4} \cdot$ Lanny J. Rosenwasser $^{5}$. Lance E. Rodewald ${ }^{6}$. Anne Eng Neo Goh ${ }^{7}$. Eitan Kerem ${ }^{8} \cdot$ Chris O'Callaghan $^{9} \cdot$ T. Bernard Kinane $^{10}$. Basil Elnazir ${ }^{11}$. Rina Triasih ${ }^{12}$. Rosemary Horne ${ }^{13}$. Anne B. Chang ${ }^{14}$. Jim Buttery ${ }^{15} \cdot$ Ruth A. Etzel $^{16} \cdot$ Kazunobu Ouchi $^{17}$. Hilary Hoey ${ }^{18}$. Varinder Singh ${ }^{19}$. Genesis C. Rivera ${ }^{20}$. Spencer S. $\mathrm{Li}^{21} \cdot$ Yu Guan ${ }^{21}$ on behalf of the Global Pediatric Pulmonology Alliance (GPPA) Council · Ling Cao ${ }^{22} \cdot$ Yue-Jie Zheng ${ }^{3} \cdot$ Lu-Zhao Feng ${ }^{23} \cdot$ Wu Zhong ${ }^{24} \cdot$ Zheng-De Xie $^{25}$. Bao-Ping Xu ${ }^{1}$. Rong-Jun Lin ${ }^{26}$. Gen Lu ${ }^{27}$. Qiang Qin ${ }^{1}$. Chun-Mei Zhu ${ }^{22}$. Su-Yun Qian ${ }^{28}$. Gang Liu ${ }^{29}$. Cheng-Song Zhao ${ }^{29}$. Zhuang Wei ${ }^{30}$. Yu-Hong Zhao ${ }^{31}$ on behalf of the Global Pediatric Pulmonology Alliance (GPPA) Expert Panel on Infectious Diseases \& COVID-19

\section{Gary Wing Kin Wong}

wingkinwong@cuhk.edu.hk

$\bowtie$ Lanny J. Rosenwasser

lrosenwasser334@gmail.com

1 China National Clinical Research Center of Respiratory Diseases, Department of Respiratory Medicine of Beijing Children's Hospital, Capital Medical University, National Center for Children's Health, Beijing, China

2 Pediatrics and Child Health Research Institute, Central Clinical Hospital, Russian Academy of Sciences, Moscow, Russian Federation

3 Department of Respiratory, Shenzhen Children's Hospital, Shenzhen, China

4 Chinese University of Hong Kong, Hong Kong SAR, China

5 UMKC School of Medicine, Kansas City, MO, USA

6 National Immunization Program, Chinese Center for Disease Control and Prevention, Beijing, China

7 K Women's and Children's Hospital, Singapore, Singapore

8 Department of Pediatrics, Hadassah Hebrew University Medical Center, Jerusalem, Israel

9 UCL Great Ormond Street Institute of Child Health and Great Ormond Street Hospital (GOSH) BRC, University College London, London, UK

10 Massachusetts General Hospital for Children, Boston, MA, USA

11 Children's Health Ireland and Trinity College Dublin, Dublin, Ireland

12 Department of Paediatric, Faculty of Medicine, Public Health and Nursing, Universitas Gadjah Mada/Dr. Sardjito Hospital, Yogyakarta, Indonesia

13 Monash University, Melbourne, Australia
14 Queensland Children's Hospital, Brisbane, Australia

15 Monash Children's Hospital, Menzies School of Health Research, Melbourne, Australia

16 George Washington University, Washington, DC, USA

17 Department of Pediatrics, Kawasaki Medical School, Kurashiki-City, Japan

18 Department of Pediatrics, University of Dublin Trinity College, Dublin, Ireland

19 Lady Hardinge Medical College and Assoc Kalawati Saran Children's Hospital, New Delhi, India

20 Center for Medical and Allied Health Sciences, New Era University, Quezon City, the Philippines

21 Global Pediatric Pulmonology Alliance, Hong Kong SAR, China

22 Department of Respiratory, the Children's Hospital Affiliated to the Capital Institute of Pediatrics, Beijing, China

23 School of Population Medicine and Public Health, Chinese Academy of Medical Sciences/Peking Union Medical College, Beijing, China

24 National Engineering Research Center for the Emergency Drug, Beijing, China

25 National Clinical Research Center for Respiratory Diseases, Laboratory of Infection and Virology, Beijing Pediatric Research Institute, Beijing Children'sHospital, Capital Medical University, National Center for Children's Health, Beijing, China 
26 The Affiliated Hospital of Qingdao University, Qingdao, China

27 Department of Respiratory, Guangzhou Women and Children's Medical Center, Guangzhou Medical University, Guangzhou, China

28 Pediatric Intensive Care Unit, Beijing Children's Hospital, Capital Medical University,

National Center for Children's Health,

Beijing, China
29 Department of Infectious Diseases, Beijing Children's Hospital, Capital Medical University, National Center for Children's Health, Beijing, China

30 Department of Health Care, Beijing Children's Hospital, Capital Medical University, National Center for Children's Health, Beijing, China

31 Department of Respiratory Medicine, Beijing Children's Hospital, Capital Medical University, National Center for Children's Health, Beijing, China 